\title{
El capital social en universitarios de Cartagena. Confianza, acción colectiva, empoderamiento y acción política*
}

\author{
Rocío M. González' \\ Jorge Palacio ${ }^{2}$ \\ Universidad del Norte (Colombia)
}

Recibido: junio 3 de 2016. Revisado: junio 25 de 2016. Aceptado: agosto 2 de 2016

Referencia formato APA: González, R., \& Palacio, J. (2016). El capital social en universitarios de Cartagena. Confianza, acción colectiva, empoderamiento y acción política. Rev. Guillermo de Ockham, 14(2), 121-130. doi: http://dx.doi.org/10.21500/22563202.2609

\section{Resumen}

El estudio indagó las características del capital social en universitarios $(\mathrm{N}=353)$, con base en la confianza, la acción colectiva, el empoderamiento y la acción política, mediante el cuestionario integrado para la medición del capital social (Inquesoc) del Banco Mundial. El comportamiento predominante es no confiar en la mayoría de las personas, entre las que se cuentan funcionarios del gobierno local y nacional y quienes conforman las relaciones en el barrio, y sí en profesores y profesionales de la salud. La solidaridad se refleja en la construcción de relaciones de intercambio recíprocas, mientras las relaciones colectivas son débiles para el trabajo conjunto y muestran desinterés en un bien colectivo que les haga movilizar. Se evidenció una disminución para reconocerse como sujeto activo y de cambio, y poco compromiso en aspectos de la vida política. Estos hallazgos son una contribución significativa en cuanto indicadores de baja asociatividad, conciencia política y empoderamiento colectivo, lo que hace que se vea disminuido el capital social.

Palabras clave: Capital social, confianza, acción colectiva, empoderamiento, acción política, universitarios, Cartagena

\section{The Social Capital in University of Cartagena: Trust, Collective Action, Empowerment and Political Action}

\section{Abstract}

The study examined the characteristics of social capital in university students ( $\mathrm{N}=353)$ from the Trust, Collective Action, Empowerment and Political Action, through the integrated measurement of social capital (INQUESOC) World Bank Questionnaire instrument. The predominant behavior is not trust most people, local officials and national government, extending these levels of distrust to relationships in the neighborhood, while relying more on teachers

\footnotetext{
* Este artículo de investigación científica deriva del proyecto El capital social de los universitarios en Cartagena, desde la confianza, la acción colectiva, el empoderamiento y la acción política (2014-2015), presentado como requisito para optar por el título de Magíster en Desarrollo Social de la Universidad del Norte.

1. Trabajadora Social. Especialista en Administración de programas de Desarrollo Social de la Universidad de Cartagena. Candidata a magíster en Desarrollo Social de la Universidad del Norte, Barranquilla (Colombia). Docente universitaria en la Corporación Universitaria Rafael Núnez, Cartagena. Correo electrónico: rocio.gonzalez@curnvirtual.edu.co

2. Ph.D. en Psicología y en Ciencias del Comportamiento y de las Prácticas Sociales por la Universidad de París X, Nanterre. Docente de la Maestría en Desarrollo Social e investigador del Centro de Investigaciones en Desarrollo Humano (Cidhum), de la Universidad del Norte, Barranquilla (Colombia).Correo electrónico: jpalacio@uninorte.edu.co
} 
and health professionals. Solidarity is reflected in building relationships of mutual exchange. Collective relations are weak for the whole and lack of a collective good that makes them mobilize work. A decrease to be recognized as active and change the subject, and little commitment on aspects of political life was evident. These findings add a significant contribution in terms of indicators of low associativity, political awareness and collective empowerment, which makes it look diminished Social Capital.

Keywords: Social capital, trust, collective action, empowerment, political action, university, Cartagena

\section{A capital social em universitarios de Cartagena. Confiança, ação coletiva, capacitação e ação política \\ Resumo}

O estudo analisou as características de capital social da universidade com base na confiança, ação coletiva, capacitação e ação política através o questionário integrado para a medição do capital social (inquesoc) do Banco Mundial. O comportamento predominante de uma amostra de 353 estudantes- é não confiar na maioria das pessoas, entre as que se contan funcionários do governo local e nacional e que tornar as relaçôes no bairro, e sim em professores e profissionais de saúde. A Solidariedade é refletida na construção de relaçôes de troca mútua , enquanto as relações coletivas são fracos para o trabalho em conjunto e mostram desinteresse em um bem coletivo que os torna movilizar. Se evidenciou uma diminuição para ser reconhecido como sujeto ativo e de mudança e pouco compromisso en aspectos da vida política. Estes resultados são uma contribuição significativa como indicadores de baixo associatividade, consciência política e empoderamento coletivo, o que faz com que pareça diminuído o capital social.

Palavras-chave: Capital social, confiança, ação coletiva, empoderamento, açáo política, universitarios, Cartagena

\section{Introducción}

El capital social cobra sentido toda vez que permite el análisis de las características de la vida social traducidas en normas de reciprocidad, redes, asociatividad, confianza y compromiso cívico que mejoran la eficacia de la sociedad y facilitan la acción coordinada (Putnam, 1994); En Cartagena se presentan contextos sociales muy diferentes, en los cuales la falta de civismo, la ingobernabilidad y la desconfianza institucional se traducen en un bajo capital social. Además, se han incrementado la incertidumbre administrativa y la desconfianza en las instituciones y se observa una gran brecha entre los valores y las necesidades que perciben los gobernantes respecto de los ciudadanos que se supone deben dirigir. Sin embargo, esta es una hipótesis que se debe probar y debemos empezar por explorar en este documento el capital social en los universitarios que, entre otras, ha sido un tema de estudio poco investigado en Cartagena, pero con un enorme potencial toda vez que aquellos asumirán un posicionamiento en la vida con formas particulares de ejercer su responsabilidad y su compromiso social frente a la construcción de nuevas ciudadanías y prácticas políticas de la ciudad. De allí surge la necesidad de formular la siguiente pregunta: ¿cuál es el capital social en universitarios de Cartagena?
Referirse a capital social es entenderlo como un recurso que permite afrontar las crisis y los desastres y recuperarse de ellos mediante la cooperación mutua para el logro de objetivos que individualmente serían inalcanzables (Coleman, 1990). Las crisis hacen pensar en las consecuencias generadas en el contexto social por la escasa participación cívica, la poca confianza institucional y el bajo empoderamiento de las acciones gubernamentales y cívicas (Correa, 2009; Pinilla E., 2007; Escobar, Quintero, Arango \& Hoyos, 2004). Las interacciones entre el capital social y las instituciones formales del Estado son muy complejas, lo que invita a entender y comprender las manifestaciones sociales de los colectivos y su forma de actuación frente a la construcción de sociedades más organizadas, cooperativas y justas. El capital social desempeña un papel importante en el estímulo de la solidaridad mediante acciones colectivas, el uso comunitario de recursos (Baas, 1997) y una mayor participación en los asuntos públicos (Lechner, 2000; Hurtado, García, \& Copete, 2011). Implica inferir en ese grado de confianza, de normas de comportamiento cívico, consenso moral y niveles de asociatividad, representado en un sistema de redes que construyen los sujetos de una sociedad, generando así riqueza y fortaleciendo el tejido social, basados en el cumplimiento de las normas y en la confianza y la capacidad de trabajar en grupos u organizaciones a 
fin de lograr objetivos comunes (Putnam, 1994; Londońo $\&$ Botero, 2013). La propuesta conceptual planteada por el Banco Mundial, contempla que las instituciones, las relaciones, las normas y las interacciones sociales de una sociedad, facilitan el desarrollo económico y la democracia (Kliksberg \& Rivera, 1998). Es así como la capacidad de las personas para trabajar en grupo con base en valores compartidos, su conciencia cívica y su sentido ético y solidario, son elementos que aseguran un clima de confianza social y fortalecimiento institucional como garantía de la gobernabilidad democrática, todo ello con base en la actitud ciudadana para aceptar esos cambios y asumirlos activamente.

La situación que atraviesa Cartagena y las transformaciones que debe generar la academia son aspectos de primera importancia, ya que las universidades deben ser vistas como entornos donde se desarrolla la vida de personas jóvenes y adultas que han logrado acceder a un modelo incluyente (Chapela \& Jarillo, 2004; Margulis \& Urresti, 1998; Altbach, 2008; Naval, 2008; Egerton, 2002), académico y orientado a la formación para el desempeño de una profesión y el ejercicio de construcción social (Mark \& Plater, 2004). Este contexto se ha estructurado a partir de imaginarios que contemplan al universitario como alguien con capacidad para aportar a la sociedad en virtud de su potencial transformador basado en su saber, sin soslayar el sentido social y humano que la sociedad demanda (Murcia, 2008; Mayorga, 2012).

Por otra parte, en los estudios encontrados en la ciudad de Cartagena, no hay una exploración del nivel de capital social de los estudiantes, razón por la cual se considera pertinente hacer una aproximación al problema para tener mayor información sobre la confianza, la acción colectiva, el empoderamiento y la acción política. Esto en la medida en que los jóvenes y universitarios en general son un activo social que construyen o fortalecen la democracia y las formas de ciudadanía para bien de la sociedad cartagenera.

\section{Metodología}

Se llevó a cabo una investigación cuantitativa con un diseño descriptivo (Hernández, Baptista, \& FernándezCollado, 2008), a partir de una población conformada por estudiantes de la Corporación Universitaria Rafael Núnez de la ciudad de Cartagena, en disciplinas de las ciencias de la salud, las ciencias sociales y humanas, las ciencias administrativas y contables y la ingeniería de sistemas. De allí se seleccionaron 353 universitarios por un muestreo probabilístico estratificado y apoyado con el programa STATS (margen de error $>5 \%$, y nivel de confianza $95 \%$ ) (Tabla 1).

Tabla 1

Muestreo probabilistico estratificado de los estudiantes por disciplinas

\begin{tabular}{lccc}
\hline \multicolumn{1}{c}{ Programas } & $\begin{array}{c}\text { Número de } \\
\text { estudiantes }\end{array}$ & $\begin{array}{c}\text { Proporción (\%) } \\
\text { de la muestra }\end{array}$ & $\begin{array}{c}\text { Muestra por } \\
\text { programa }\end{array}$ \\
\hline Medicina & 904 & $21 \%$ & 74 \\
\hline Odontología & 304 & $7 \%$ & 25 \\
\hline Bacteriología & 171 & $4 \%$ & 14 \\
\hline Enfermería & 501 & $12 \%$ & 42 \\
\hline Inst. quirúrgica & 177 & $4 \%$ & 14 \\
\hline Contaduría & 241 & $6 \%$ & 21 \\
\hline $\begin{array}{l}\text { Administración de } \\
\text { empresas }\end{array}$ & 120 & $3 \%$ & 11 \\
\hline Trabajo social & 492 & $12 \%$ & 42 \\
\hline Derecho & 762 & $18 \%$ & 64 \\
\hline $\begin{array}{l}\text { Lic. en pedagogía } \\
\text { infantil }\end{array}$ & 463 & $11 \%$ & 39 \\
\hline Ing. de sistemas & 77 & $2 \%$ & 7 \\
\hline Total & 4.212 & $100 \%$ & 353 \\
\hline Fuente: Elaboración propia. & &
\end{tabular}

Los criterios de inclusión fueron ser estudiantes de una institución de educación superior y residentes en Cartagena o municipios aledaños. Los encuestados, en su mayoría, son hombres que provienen de diferentes niveles socioeconómicos en los que predominan los estratos uno y dos.

Respondieron el cuestionario integrado para la medición del capital social (Inquesoc) del Banco Mundial (Grootaert, C., Narayan, D., Nyhan, V., \& Woolcock, M., 2002), que pasó por el siguiente proceso de validación. En primer lugar, fue sometido a una prueba piloto con un grupo de universitarios para determinar su funcionamiento y comprensión al momento de su aplicación. Se mantuvieron el equilibrio del rigor conceptual, la flexibilidad transcultural y la adaptabilidad, mediante ajustes del instrumento al lenguaje (contextualizarlo), y a la redacción u organización de las preguntas para su comprensión. Se mantuvo un número de hojas reducido para evitar respuestas distantes de la realidad. En segundo lugar, se sometió a validación por jueces expertos del Grupo de Investigaciones en Desarrollo Humano (GIdhum) y profesores de la maestría de Desarrollo Social. Está conformado por varias dimensiones, a saber: grupos y redes (veintidós preguntas), confianza y solidaridad (seis preguntas), acción colectiva y cooperación (cinco preguntas), información y comunicación (cinco preguntas), cohesión e inclusión social (veinticinco preguntas) y empoderamiento y acción política (doce preguntas). Se retoman de estas las variables acción colectiva, confianza, empoderamiento y acción po- 
lítica, de interés para la investigación y se capta el grado de confianza de los universitarios en los siguientes aspectos: grupos de personas; sus comunidades; ayuda de miembros de sus comunidad o red de apoyo en caso de necesidad; participación y apoyo colectivo; control sobre las decisiones que afectan directamente la vida cotidiana; forma como perfeccionan este control (petición a funcionarios del gobierno, participación en reuniones públicas, participación en elecciones); percepción de la honestidad de los funcionarios del Gobierno, y extensión de la corrupción. Se tienen en cuenta, además, las consideraciones éticas de la información, su procedimiento, el bajo riesgo y los beneficios de investigación. Todos los estudiantes fueron informados sobre el instrumento motivo del estudio y la confidencialidad. Su aprobación para participar se dio mediante la firma del consentimiento informado.

\section{Resultados}

De la población de estudio, el 70,5 \% son hombres (media $=19,8$ años en hombres y 20,2 en mujeres). El $87,8 \%$ son solteros, de los cuales el $90,9 \%$ se dedica solo al estudio. El 59,2 \% es oriundo de Cartagena, el 15,6 \% de otros municipios del departamento de Bolívar y un $25,2 \%$ de otros departamentos del país, especialmente de la Costa Atlántica. Viven en barrios que pertenecen en su mayoría a los estratos uno y dos (68 \%).
Los resultados refieren tres dimensiones del capital social, que se explicitan a continuación:

\section{Confianza y solidaridad}

Se encontró representado con $53,8 \%$ de desconfianza y de $46,2 \%$ de confianza (Tabla 2 ).

A pesar de que en las mujeres hay una tendencia a no confiar en nadie $(71,6 \%)$, no se encontró asociación estadística entre el sexo, la procedencia o estrato y la confianza $\left(X^{2}=0,214 ;\right.$ Sig asintótica bilateral $=6,43 ; X^{2}=0,268$, Sig asintótica bilateral $=0,874 ; \mathrm{X}^{2}=5,725 ;$ Sig asintótica bilateral $=0,334$, respectivamente)

Esta desconfianza generalizada es visible en procedentes de Cartagena y de diferentes estratos. Estos niveles de desconfianza personal se ven reflejados en el hecho de que el $62,1 \%$ considera que en su barrio se debe estar alerta o alguien se aprovechará.

Otro hallazgo significativo estiba en que el $98 \%$ de las personas en su barrio está dispuesta a ayudar cuando es necesario; el resto asume una posición neutra o de desacuerdo. Si se hace una comparación global entre las distintas manifestaciones de confianza en diferentes tipos de personas, se observa que los estudiantes universitarios de Cartagena confían en una proporción mayor en profesores $(9,1 \%)$ y enfermeras/médicos $(8,5 \%)$, y desconfían en extraños (79,3 \%) y en los partidos políticos $(54,4 \%)$ (Tabla 3$)$.

Tabla 2

Confianza según sexo, procedencia y estrato

\begin{tabular}{|c|c|c|c|c|c|c|c|}
\hline & & Se puede confiar & $\%$ & $\begin{array}{c}\text { No se puede } \\
\text { confiar en nadie }\end{array}$ & $\%$ & $\mathbf{X}^{2}$ & $\begin{array}{c}\text { Sig asintótica } \\
\text { bilateral }\end{array}$ \\
\hline \multirow{3}{*}{ Sexo } & Hombre & 50 & $30,7 \%$ & 54 & $28,4 \%$ & 0,214 & 0,643 \\
\hline & Mujer & 113 & $69,3 \%$ & 136 & $71,6 \%$ & & \\
\hline & & 163 & & 190 & & & \\
\hline \multirow{4}{*}{ Procedencia } & Cartagena & 98 & $60,12 \%$ & 111 & $58,42 \%$ & 0,268 & 0,874 \\
\hline & Municipios de Bolívar & 26 & $15,95 \%$ & 29 & $52,73 \%$ & & \\
\hline & Otros departamentos & 39 & $23,93 \%$ & 50 & $73,53 \%$ & & \\
\hline & & 163 & & 190 & & & \\
\hline \multirow{7}{*}{ Estrato } & Uno & 56 & $34,4 \%$ & 55 & $28,9 \%$ & 5,725 & 0,334 \\
\hline & Dos & 61 & $61,0 \%$ & 68 & $35,8 \%$ & & \\
\hline & Tres & 34 & $20,9 \%$ & 45 & $23,7 \%$ & & \\
\hline & Cuatro & 10 & $6,1 \%$ & 13 & $6,8 \%$ & & \\
\hline & Cinco & 1 & $0,6 \%$ & 8 & $4,2 \%$ & & \\
\hline & Seis & 1 & $0,6 \%$ & 1 & $0,5 \%$ & & \\
\hline & & 163 & & 190 & & & \\
\hline
\end{tabular}

Fuente: Elaboración propia. 
Tabla 3

Nivel de confianza por grupos de personas

\begin{tabular}{|c|c|c|c|c|c|c|c|c|c|c|c|c|c|c|c|c|}
\hline & \multicolumn{2}{|c|}{$\begin{array}{l}\text { Personas de } \\
\text { su grupo } \\
\text { étnico o } \\
\text { lingüístico/ } \\
\text { raza/ tribu }\end{array}$} & \multicolumn{2}{|c|}{$\begin{array}{c}\text { Funcionario } \\
\text { del gobierno } \\
\text { local }\end{array}$} & \multicolumn{2}{|c|}{$\begin{array}{c}\text { Funcionario } \\
\text { del gobierno } \\
\text { central }\end{array}$} & \multicolumn{2}{|c|}{$\begin{array}{l}\text { Partidos } \\
\text { políticos }\end{array}$} & \multicolumn{2}{|c|}{ Policía } & \multicolumn{2}{|c|}{$\begin{array}{l}\text { Enfermeras y } \\
\text { médicos }\end{array}$} & \multicolumn{2}{|c|}{ Profesores } & \multicolumn{2}{|c|}{ Extrańos } \\
\hline & Fr & $\%$ & $\mathrm{Fr}$ & $\%$ & Fr & $\%$ & $\mathrm{Fr}$ & $\%$ & $\mathbf{F r}$ & $\%$ & $\mathrm{Fr}$ & $\%$ & $\mathrm{Fr}$ & $\%$ & $\mathrm{Fr}$ & $\%$ \\
\hline Muy poco & 83 & 23,5 & 166 & 47,0 & 166 & 47,0 & 192 & 54,4 & 93 & 26,3 & 23 & 6,5 & 17 & 4,8 & 280 & 79,3 \\
\hline Poco & 81 & 22,9 & 117 & 33,1 & 117 & 33,1 & 97 & 27,5 & 106 & 30,0 & 46 & 13,0 & 36 & 10,2 & 48 & 13,6 \\
\hline $\begin{array}{l}\text { Ni mucho ni } \\
\text { poco }\end{array}$ & 143 & 40,5 & 66 & 18,7 & 66 & 18,7 & 55 & 15,6 & 117 & 33,1 & 91 & 25,8 & 86 & 24,4 & 15 & 4,2 \\
\hline Mucho & 39 & 11,0 & 3 & 0,8 & 3 & 0,8 & 7 & 2,0 & 34 & 9,6 & 163 & 46,2 & 182 & 51,6 & 5 & 1,4 \\
\hline $\begin{array}{l}\text { En una propor- } \\
\text { ción muy grande }\end{array}$ & 7 & 2,0 & 1 & 0,3 & 1 & 0,3 & 2 & 0,6 & 3 & 0,8 & 30 & 8,5 & 32 & 9,1 & 5 & 1,4 \\
\hline Total & 353 & 100 & 353 & 100 & 353 & 100 & 353 & 100 & 353 & 100 & 353 & 100 & 353 & 100 & 353 & 100 \\
\hline
\end{tabular}

Fuente: Elaboración propia.

En perspectiva similar, consideran que en los últimos cinco años el nivel de confianza en su barrio ha mejorado $(24,6 \%)$, empeorado $(26,1 \%)$, o se ha mantenido $(49,3 \%)$, reflejo de un debilitamiento en la confianza que imposibilita la movilización, el liderazgo y el sentido social hacia un desarrollo social de sus comunidades. En relación con un proyecto de la comunidad que no lo beneficia directamente, pero tiene beneficios para otras personas de su barrio, el estudiante contribuiría de a siguiente manera: con tiempo el 81,6\%; con dinero el 55,2\% (Tabla 4).

Tabla 4

Contribución con tiempo o dinero a un proyecto

\begin{tabular}{lcc}
\hline & Frecuencia & $\begin{array}{c}\text { Porcentaje } \\
\%\end{array}$ \\
\hline No contribuye con tiempo & 65 & 18,4 \\
\hline Contribuye con tiempo & 288 & 81,6 \\
\hline No contribuye con dinero & 158 & 44,8 \\
\hline Contribuye con dinero & 195 & 55,2 \\
\hline Total & 353 & 100,0 \\
\hline
\end{tabular}

Fuente: Elaboración propia.

\section{Acción colectiva y cooperación}

$\mathrm{Al}$ respecto, el 70,8 \% expresó que el año anterior no trabajó con personas de su barrio para beneficio de la comunidad, evidenciando con ello baja asociación para el trabajo comunitario y bajo compromiso hacia sus comunidades, lo cual coincide con la tendencia mostrada en el estudio (Hurtado, García, \& Copete, 2011), en el que las variables críticas de capital social en la ciudad de Cartagena son solidaridad general, solidaridad en las relaciones horizontales, solidaridad vertical y actividades sociales con los vecinos, contribuyendo de esta manera que el capital social en Cartagena sea bajo. De la tendencia encontrada en relación con la contribución más con tiempo que con dinero en un proyecto para la comunidad, la proporción de estas personas que colaboran ascendería a menos de la mitad (35,1\%). Es decir, que dicha contribución en lo que atañe al beneficio común y colectivo es muy baja. Respecto de la cooperación, definida como las actitudes de las personas frente a aspectos de interés colectivo y público (Kliksberg \& Rivera, 1998), se encontró que para la búsqueda de soluciones a problemas concretos las personas colaborarían $(73,3 \%$ ) y la probabilidades de que se organicen para ayudar a la comunidad ante un hecho desafortunado es del 77,6 \%.

No se encontró asociación estadística entre el sexo y la acción colectiva o cooperación $\left(\mathrm{X}^{2}=1,429\right.$; Sig asintótica bilateral $=0,232 ; X^{2}=6,413 ;$ Sig asintótica bilateral $=$ $0,170)$.

\section{Empoderamiento y acción política}

En esta esfera, el $91 \%$ considera que es feliz. Asimismo, se da un significativo reconocimiento de su área individual y se mantiene un control eficiente sobre las decisiones $(81,9 \%)$, por encima del sentimiento de no control que se percibe $(18,2 \%)$. Consideran que tienen derechos y poder de cambiar el curso de su vida ( $92 \%)$, en comparación con quienes no lo ven así. El concepto sobre la influencia para los cambios en sus contextos comunitarios y hacer que su barrio sea un mejor lugar para vivir, se ve disminuido en los universitarios, toda vez que el 70,2 \% considera que tienen poca o ninguna influencia. Adicionalmente, se encontró que el 74,2 \% de las personas en su barrio se reunían para hacer peticiones en conjunto a funcionarios del Gobierno o a líderes políticos acerca de 
alguna cuestión que beneficiara a la comunidad. En esta dirección, se logró una gestión exitosa en el teniendo éxito en el $47,8 \%$ de los casos.

En lo tocante a las acciones llevadas a cabo en el último año, los estudiantes universitarios, en su mayoría, no participaron ni asistieron a actividades asociadas con la vida pública (Figura 1). En las últimas elecciones para alcalde y presidente, el 58,4\% de los estudiantes no votaron; sin embargo, a la hora de elegir a un candidato representativo de un grupo étnico diferente al de ellos, respondieron que sí lo elegiría $(84,7 \%)$. En relación con la confianza, consideran más honestos a los profesores $(54,3 \%$. $r$ de Pearson= 0,393), y al personal del sector salud $(51,2 \%$. $r$ de Pearson= 0,373). Además, es coincidente el grado de desconfianza con el de deshonestidad, de la siguiente manera: en relación con funcionarios del Gobierno: 60,3\%. ( $\mathrm{r}$ de Pearson=0,295); funcionarios de la policía: 43,3\% ( $\mathrm{r}$ de Pearson=0,462), y líderes comunitarios: $28,6 \%$ ( $\mathrm{r}$ de Pearson=0,160). En comparación con los índices de cinco años atrás, un 50,7 \% del estudiantado considera que la honestidad del Gobierno local se ha deteriorado; un $35,7 \%$ que se ha mantenido y un $13,6 \%$ que ha mejorado (no se encontró asociación estadística entre el control en la toma de decisiones y la participación en los procesos electorales. $X^{2}=2,341 ;$ Sig $=0,673$ ).

\section{Figura 1}

Actividades 0 acciones en que ha participado en el último año

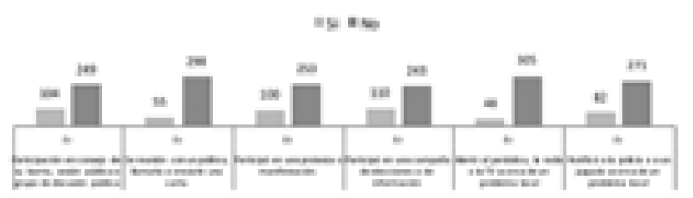

Fuente: Elaboración propia.

\section{Discusión}

Tal como lo plantea Putnam, el capital social es concebido con base en la acumulación de varios tipos de activos, como la confianza, el comportamiento cívico y el nivel de asociatividad, aumentando con ello la probabilidad de un comportamiento cooperativo, mutuo y beneficioso para las personas que lo poseen y para la sociedad en cuanto fortalece el tejido social (Aguirre, 2012).

\section{Confianza y solidaridad}

Entendidas como el resultado de las interacciones con personas que demuestran en la experiencia acumulada que responderán con quid pro quo a un acto de generosidad, alimentando así un vínculo que combina la aceptación del riesgo con un sentimiento de afectividad o identidad ampliada (Arriagada, 2001). La tendencia de los universitarios es no confiar en la mayoría de las personas, lo cual está en similitud con los resultados de otras investigaciones (De la Hoz \& Madariaga, 2011; Herrera \& Madariaga, 2013; Vergara \& Ramos, 2010; Gutiérrez, y otros, 2001).

En este grupo de personas, las que presentan un nivel de desconfianza más alto son las mujeres, lo que se traduce en un nivel de confianza más visible en los hombres. Estos niveles se extienden hasta las relaciones forjadas en el barrio donde habitan; empero, consideran que se debe estar alerta ya que alguien puede aprovecharse, lo que no es óbice para considerar que la mayoría de sus vecinos están dispuestos a ayudar cuando sea necesario. Es decir, a pesar de no confiar en el otro, se es consciente de la necesidad de colaboración siempre que esta se requiera.

Así mismo, se encontraron manifestaciones de desconfianza hacia extraños, partidos políticos y funcionarios de los gobiernos local y central. Esto pone de manifiesto que no creen en las instituciones ni en los partidos por su falta de trasparencia. Esta desconfianza puede haber sido provocada por la situación política y social que atraviesa Cartagena durante los últimos ańos, concretada en ingobernabilidad, cambios políticos de tipo clientelista, corrupción, tensión social e inseguridad, entre otras (Cartagena como vamos- CCV, 2014). Lo primero que se destaca en el discurso de todos los colectivos es una imagen negativa del Estado y de quienes buscan llegar a él: los políticos.

En esa medida, la imagen que se tiene de los partidos políticos también es negativa, fruto de la distancia entre estos y los jóvenes (Pinilla \& Henao, 2009), lo que coincide con los resultados relacionados con la casi total desconfianza en las instituciones que guardan relación con el poder público (funcionarios y políticos) (Olmos \& Amar, 2006). Bajo esta perspectiva, las universidades desempeñan un papel de suma importancia ante la inercia eventual del potencial existente y es menester que se trabaje, mediante políticas académicas y otros medios en virtud de sus fines, en pos de su fortalecimiento y desarrollo. Los mayores niveles de confianza se depositan en los profesionales de la salud y los profesores, en cuanto representan una figura de respeto y reciprocidad tanto en los procesos educativos como de la salud. Lo anterior invita a las instituciones y a sus representantes a diseñar nuevas estrategias para que el universitario conozca y se reconozca en ellos, como modelos de solidaridad y apoyo social.

La población de universitarios considera que en los últimos cinco años el nivel de confianza en su barrio se ha 
mantenido, lo que indica un estancamiento en los niveles de desconfianza si se tiene en cuenta que sus respuestas denotaban la necesidad de permanecer alerta y desconfiar del otro. La mayoría considera que las personas de su barrio mantienen una situación que apunta a una mezcla de actitudes entre llevarse bien y llevarse mal; es decir, no hay una proximidad completa que permita crear lazos de confianza con los demás.

Otro elemento en esta dimensión sobre la cual se construye el capital social es la inversión en tiempo y esfuerzo, y en esta dirección es clara la preferencia a contribuir con tiempo, en un proyecto. Cuando estos recursos básicos (intangibles) son aprovechados voluntariamente para estructurar relaciones de intercambio recíprocas o cooperativas, se tiene un capital social. Los jóvenes se agrupan con base en objetivos concretos cuando se trata de expresar solidaridad ante la vulneración de los derechos o de luchar por la paz. Es, entonces, cuando se manifiestan el capital social y sus posibilidades de contribuir en proyectos que buscan equidad y justicia social (Aguirre, 2012). Se generan, sin embargo, dos fenómenos que provocan la poca participación política de la juventud: la ausencia de un plan estatal que defina el curso la sociedad y la pérdida del poder socializador de las instituciones (Cubides, Guerrero, Aladier, Hurtado, \& Restrepo, 2007).

Podemos concluir que el capital de los universitarios en Cartagena, en esta dimensión de confianza y solidaridad, reflejan valores de solidaridad, pero sigue débil la confianza social e institucional, especialmente aquella que representa la figura estatal muy vinculada a falta de transparencia, respeto y lealtad, lo que resulta en una visión negativa de los dirigentes.

\section{Acción colectiva y cooperación}

Explica la conducta de un individuo o la acción de un colectivo con el fin de prever no solo lo que harían aisladamente, sino cuándo y actuarán conjuntamente con un mismo propósito. En este sentido, un porcentaje alto de universitarios en Cartagena no trabajaron en el último año conjuntamente con otras persona en su barrio, en proyectos que generaran un beneficio común; es decir, no hay un interés compartido que los haga movilizarse. Paradójicamente, a pesar de no participar conjuntamente con otros, siempre existirá la tentación de esperar que sea otro quien se movilice y obtener, igualmente, los beneficios en caso de que la acción tenga éxito.

Esto podría explicar que los estudiantes universitarios no compartan con sus vecinos un interés común que los lleve a actuar conjuntamente. Sin embargo, para una parte menor el valor de la acción colectiva es superior al beneficio individual que les puede reportar, por ende puede suceder que la movilización sea más reducida de lo que podría esperarse, pero impregnada de una alta valoración. Lo anterior indica que la acción colectiva, como indicador de capital social en los universitarios de Cartagena, está disminuida.

Desde la perspectiva del estudiante universitario en las comunidades en las que interactúa, las personas contribuyen más con tiempo que con dinero en un proyecto, lo que se convierte en en elemento favorable para el trabajo comunitario y aumenta la probabilidad de que un gran porcentaje de personas de la comunidad cooperen para resolver los problemas. La medición del capital social en el 2011 y el informe de la ciudad de Cartagena muestran un resultado similar, pues en ellos se demostró que los cartageneros pueden esperar ayuda por parte de sus vecinos. Esto refleja un cambio de actitud de los ciudadanos como actores responsables que no esperan que el Estado se encargue de las actividades del bien colectivo, lo cual corrobora que la cooperación es una conducta que va más allá del interés propio (preferencias sociales, normas de reciprocidad, comportamientos altruistas) y se torna en un aspecto importante para el desarrollo en el sentido que reconstruye el trabajo cooperativo y el interés en el otro y proponen salir adelante si existe un objetivo común.

En la organización de la comunidad para ayudar ante un evento desafortunado, se da una notable percepción en el sentido de que pueden esperar ayuda del otro, lo que patentiza el vínculo y la confianza social que se genera en los colectivos comunitarios. La cooperación y organización en grupos en los cuales se inscribe el estudiante universitario, constituyen un factor fundamental de capital social. El compromiso cívico caracteriza una comunidad cívica; es decir, una sociedad en la que los ciudadanos están dispuestos a la confianza y a la solidaridad y manifiestan un interés por los asuntos públicos (Urteaga, 2013), siempre y cuando se den elementos de confianza social y un fortalecimiento institucional como garantía de la gobernabilidad democrática.

\section{Empoderamiento y acción política}

Los estudiantes universitarios en Cartagena se consideran muy felices a pesar de las condiciones socialmente difíciles que se viven en la ciudad y de su inconformismo y desconfianza en las personas e instituciones. La mayor parte de los estudios han medido el nivel de bienestar subjetivo o de felicidad y coinciden en los altos niveles de 
este sentimiento y en su persistencia en el tiempo. En un sentido más estricto, la felicidad se concibe como parte del bienestar subjetivo ya que este alude al juicio global que hacen las personas de su propia vida y esta evaluación es tanto cognoscitiva como afectiva (Gómez, Villegas, Barrera, \& Cruz, 2007).

Otro resultado de la investigación consiste en que los estudiantes universitarios manifiestan tener un fuerte control sobre aquellas decisiones que afecten sus actividades diarias y su vida personal. Ello se traduce en un empoderamiento individual, pero este no es, necesariamente, un factor que aporta al ejercicio de sus derechos políticos y ciudadanos comoquiera que a pesar de ser sujetos de derechos y poseer poder sobre sus vidas, no se observa una clara determinación de participar en los cambios sociales por la falta de credibilidad y confianza institucionales.

A pesar de esa felicidad, el control sobre sí mismos y reconocerse poseedores de derechos, los estudiantes universitarios consideran que tienen poca influencia para hacer de su barrio un mejor lugar para vivir, lo que demuestra que no se reconocen como sujetos activos y de cambio y poseedores de recursos para transformar las comunidades a las que pertenecen. Asimismo, se trasluce en sus concepciones un deterioro del concepto de comunidad como una construcción en la que el individuo tienen la posibilidad de crear sentido de identidad, fortalecerla y resignificarla como espacio para el desarrollo de capacidades deliberantes.

En otras palabras, el empoderamiento si bien es cierto incluye tanto el cambio individual como la acción colectiva, en los estudiantes universitarios de Cartagena solo llega hasta el plano individual. Esta integración con su comunidad y la colaboración en actividades u organizaciones de acciones comunitarias, generarían participación en esferas más amplias de la sociedad y se constituirían por sí mismas en un importante componente del capital social. Sin embargo, al revisar los resultados en relación con las acciones efectuadas en el último año, se observa que en su mayoría no participaron ni asistieron a actividades asociadas a la vida pública o a espacios en los que percibe una gran fuerza para el cambio democrático y el reconocimiento como ciudadanos transformadores y políticos.

Lo primero que se destaca en el discurso del colectivo es una imagen negativa del Estado y de quienes buscan llegar a él, a saber, los políticos. En esa medida, la representación que se tiene de los partidos políticos es también negativa y se manifiesta en una baja participación política en las últimas elecciones de para alcalde y presidente, lo cual es una constante en los jóvenes colombianos (Pinilla \&
Henao, 2009; Chapela \& Jarillo, 2004). Los grupos en los que confían en cuanto a su grado de honestidad, están conformados por los profesores y el personal del sector salud, y el mayor grado de desconfianza se enfoca en los colectivos que consideran más deshonestos, representados en los funcionarios del Gobierno, la Policía y los líderes comunitarios (en ese orden).

Es importante que los estudiantes universitarios comprendan que cada movimiento asumido con el propósito de conseguir un mayor control sobre sus vidas hará que broten otras necesidades sociales y políticas en las cuales deben comprometerse para impulsar un proceso que sea doblemente transformador: como agentes sociales conscientes de su transformación individual y promotores de nuevos cambios colectivos, en una ciudad necesitada de nuevas voces y acciones que fortalezcan la estructura social y ciudadana (Henao, Ocampo, Robledo, \& Lozano, 2008). Estos hallazgos añaden una contribución significativa, en cuanto puede concluir que los universitarios de Cartagena presentan indicadores de baja asociatividad, conciencia política y empoderamiento colectivo, lo que hace que se vea disminuido el capital social (Tabla 3).

Tabla 3

El capital social en universitarios de Cartagena

\begin{tabular}{|c|c|c|}
\hline \multirow[t]{3}{*}{$\begin{array}{l}\text { Dimensión } \\
\text { confianza y } \\
\text { solidaridad }\end{array}$} & $\begin{array}{l}\text { Confianza social: }(-) \\
/(+)^{*}\end{array}$ & $\begin{array}{l}\text { (-): El comportamiento predomi- } \\
\text { nante es no confiar en la mayoría } \\
\text { de las personas. } \\
(+) \text { : Reconocimiento que la mayo- } \\
\text { ría de los vecinos colaborarían al } \\
\text { necesitar ayuda. Al igual de creer } \\
\text { más en profesores y profesionales } \\
\text { de la salud. }\end{array}$ \\
\hline & $\begin{array}{l}\text { Confianza institucio- } \\
\text { nal: (-) }\end{array}$ & $\begin{array}{l}\text { Representado en la desconfianza } \\
\text { a funcionarios del gobierno local } \\
\text { y nacional. }\end{array}$ \\
\hline & Solidaridad: $(+)$ & $\begin{array}{l}\text { Construcción de relaciones de } \\
\text { intercambio recíprocas. }\end{array}$ \\
\hline \multirow[t]{2}{*}{$\begin{array}{l}\text { Dimensión ac- } \\
\text { ción colectiva y } \\
\text { cooperación }\end{array}$} & Acción colectiva: (-) & $\begin{array}{l}\text { Relaciones colectivas débiles para } \\
\text { el trabajo conjunto. No hay interés } \\
\text { compartido de un bien colectivo } \\
\text { que los movilice. }\end{array}$ \\
\hline & Cooperación: (+) & $\begin{array}{l}\text { Representado en la posibilidad de } \\
\text { ayuda y actuación conjunta para } \\
\text { resolver problemas. }\end{array}$ \\
\hline \multirow{3}{*}{$\begin{array}{l}\text { Dimensión } \\
\text { empodera- } \\
\text { miento y } \\
\text { acción política }\end{array}$} & $\begin{array}{l}\text { Empoderamiento } \\
\text { individual: }(+)\end{array}$ & $\begin{array}{l}\text { Control en decisiones de su } \\
\text { vida diaria y personal (plano } \\
\text { individual). }\end{array}$ \\
\hline & $\begin{array}{l}\text { Empoderamiento } \\
\text { colectivo: }(-)\end{array}$ & $\begin{array}{l}\text { Disminución para reconocerse } \\
\text { como sujeto activo y de cambio } \\
\text { (plano colectivo). }\end{array}$ \\
\hline & Acción política: (-) & $\begin{array}{l}\text { Poco compromiso en aspectos de } \\
\text { la vida política que genere com- } \\
\text { promisos con la sociedad. }\end{array}$ \\
\hline
\end{tabular}
capital social 


\section{Conclusiones}

Esta disminución de capital social genera una desconfianza horizontal y vertical y el tejido social no se ve fortalecido ni se evidencia apropiación de sus miembros en la capacidad de trabajar juntos en grupos u organizaciones a fin de lograr objetivos comunes. Es, entonces, cuando el Estado debe recuperar la confianza e influir de manera más directa para la creación del capital social mediante la educación. Las instituciones educativas no transmiten simplemente capital humano sino también las reglas y normas implícitas, las cuales son asumidas por los miembros de la sociedad en la que se enmarca (Portela $\&$ Neira, 2011).

Si en la actualidad los jóvenes no comparten las formas convencionales de participación política, inferir niveles de compromiso cívico a partir de la participación política convencional sería una forma limitada de estimar el compromiso cívico (Varela, Martínez, \& Cumsille, 2015), lo que significaría ampliar los estudios en cuanto a la relación de la participación política con compromiso cívico (Putnam, 1994).

Finalmente cabe resaltar la importancia del presente estudio, dado que en Cartagena no se ha estudiado esta temática a profundidad e igualmente resultó limitante para contrastar con otros resultados, lo que invita a seguir trabajando en instrumentos más robustos y contextualizados que permitan aplicar métodos estadísticos profundidad para el análisis de la información.

\section{Referencias}

Aguirre, N. L. (2012). La confianza, los valores y el capital social en jóvenes. Hacia una comprension de la trascendencia humana. Revista de Investigaciones, 23(2), 112-119.

Altbach, P. G. (2008). Funciones compleja de las universidades en la era de la globalización. En P. Taylor (Ed.), La educación superior en el mundo: Educacion superior, Nuevos retos y roles emergentes para el desarrollo humano y social. (pp. 5-14). Madrid: Mundi-Prensa.

Arriagada, I. (2001). Capital social: potecialidades y limitaciones analíticas de un concepto. Revista CEPAl, 31, 13-29.

Baas, S. (1997). News trends in rural development and poverty alleviation: the concept of participatory institutional development. Trabajo presentado en la Bolsa Internacional Académico Conferencia sobre Agricultura Sostenible y arena de control en Gansu área del desierto, China.

Balardini, S. (2000). La participación socialy politica de los jóvenes en el horizonte del nuevo siglo. Buenos Aires: CLACSO.
Cartagena como vamos- CCV. (2014). Resultados de la encuesta de percepcion ciudadana e indicadores técnicos. Recuperado de https://goo.gl/p3TDMS

Chapela, M. D., \& Jarillo, E. C. (2004). El capital social en el futuro de la Universidad. Reencuentro, 40, 1-13.

Coleman, J. S. (1990). Foundations of social theory. MA: Harvard University Press.

Correa, M. S. (2009). Nuevas Prácticas Políticas en Jóvenes de Chile: conocimientos acumulados 2000-2008. Revista Latinoamericana de Ciencias Sociales, Niñez y Juventud, 7(2), 1379-1403.

Cubides, H., Guerrero, P., Aladier, J., Hurtado, D., \& Restrepo, A. (2007). Jóvenes, Participación política y formación democrática. Estudio comparado en Bogotá y Medellín. Nomadas, 26, 230-233.

De la Hoz, Y., \& Madariaga, C. (2011). Relación entre la inserción social y el capital social en población desplazada. (Tesis de maestría innédita). Uninorte. Barranquilla, Colombia.

Egerton, M. (2002). Higher education and civic engagement. The British Journal of Sociology, 53(4), 603-620.

Escobar, M. R., Quintero, F., Arango, A. M., \& Hoyos, D. (2004). Estado del arte del conocimiento producido sobre jóvenes 1985-2003. Bogota, DC: UNICEF.

Gómez, V., Villegas, C., Barrera, F., \& Cruz, J. E. (2007). Factores predictores de bienestar subjetivo en una muestra Colombiana. Revista Latinoamericana de Psicología, 39(2), 31-325.

Grootaert, C., Narayan, D., Nyhan, V., \& Woolcock, M. ( 2002). Cuestionario Integrado para la medición de capital social. Banco Mundial. . Washington, DC, USA.

Gutiérrez, K., Sabatier, C., Madariaga, C., Palacio, J., Abello, R., \& Amar Amar, J. (2001). El capital social como apoyo a la superación de problemas en la población desplazada por la violencia en Colombia. Investigación \& Desarrollo, $9(2), 514-535$.

Henao, J., Ocampo, A., Robledo, Á., \& Lozano, M. (2008). Los grupos juveniles universitarios y la formación ciudadana. Universitas Psychologica, 7(3), 853-867.

Hernandez, R., Baptista, P., \& Fernández-Collado, C. (2008). Metodología de la investigación. Madrid: Mc Graw Hill.

Herrera, J., \& Madariaga, C. (2013). Comisaría de Familia de Canapote: entidad que genera capital social. Rev. Guillermo de Ockham, 11(1), 49-64.

Hurtado, D., García, D., \& Copete, A. (2011). Tercera medición de Capital Social en Colombia BARCAS 2011. Informe de medición de capital social en Cartagena. Bogotá: Fundación Antonio Restrepo. 
Kliksberg, B., \& Rivera, M. (1998). Seis tesis no convencionales sobre participacion. Revista de Estudios Sociales. Red de Gobernabilidad y Desarrollo Institucional PNUD.

Lechner, N. (Enero de 2000). Nuevas Ciudadanías . Revista de estudios sociales, 5, 25-31.

Londoño, C., \& Botero, J. (2013). Aproximación al Concepto de Capital Social. Sinapsis, 5(5), 96-104.

Margulis, M., \& Urresti, M. (1998). La construcción social de la condición de juventud. Recuperado de https://goo.gl/PLLJE8

Mark, L., \& Plater, W. M. (2004). Public Work and the Academy: An Academic Administrator's Guide to Civic Engagement and Service-Learning. Bolton, MA: Anker Publishing Co.

Mayorga, P. (2012). Capital humano y capital social contexto de la calidad en la educación Superior. Revista Estudios hemisfericos y polares., 3(4), 222-242.

Murcia, N. (2008). Jóvenes universitarios y universitarias: una condición de visibilidada parente en Colombia. Revista Latinoamericana en Ciencias sociales, niñez y juventud, 6(2), 821-852.

Naval, C. (2008). Universidad y conciencia civica: algunas experiencias fructiferas. Service learning y campus compact. Concepción: Pontificia Universidad Católica de Chile.

Olmos, F., \& Amar, J. (2006). Capital social de los estudiantes de economía de las universidades en la ciudad de Barranqui- lla. (Tesis de maestría Inedita). Uninorte. Barranquilla, Colombia.

Pinilla, V. E. (2007). Significado de lo público para un grupo de jóvenes universitarios. (Tesis de Doctorado inédita). Universidad de Manizale. Manizalez, Colombia.

Pinilla, V. E., \& Henao, J. (Julio - Diciembre de 2009). Jóvenes y ciudadanías en Colombia: entre la politización social y la participación institucional. Revista Latinoamericana de Ciencias Sociales, Niñez y Juventud, 7(2), 1405-1437.

Portela, M., \& Neira, I. (2011). Determinantes del capital social en España. Recuperado de: http://2011.economicsofeducation.com/malaga2011/Neira.pdf.

Putnam, R. (1994). Para hacer que la democracia funcione. Venezuela: Editorial Galac.

Urteaga, E. (Junio de 2013). La teoría del capital social de Robert Putnam: Originalidad y carencias. Reflexión Politica, 15(29), 44-60.

Varela, E., Martínez, M., \& Cumsille, P. (2015). ¿Es la participación política convencional un indicador del compromiso cívico de los jóvenes? Universitas Psychologica, 14(2), 715730. doi: http://dx.doi.org.10.11144/Javeriana.upsy14-2.eppc

Vergara, M., \& Ramos, J. (2010). Análisis de los factores de capital social asociados a la capacidad competitiva de las empresas. (Tesis de Maestría inédita). Uninorte. Barranquilla, Colombia. 